\title{
Excitation of surface phonon polariton modes in gold gratings with silicon carbide substrate and their potential sensing
}

\section{applications}

\author{
Gaige Zheng $^{\text {a,b*, }}{ }^{\text {Linhua }} \mathrm{Xu}^{\text {a }}$, Xiujuan Zou ${ }^{\mathrm{a}}$,Yuzhu Liu ${ }^{\text {a,b }}$ \\ ${ }^{a}$ School of Physics and Optoelectronic Engineering, Nanjing University of \\ Information Science \& Technology, Nanjing 210044, China \\ ${ }^{\mathrm{b}}$ Jiangsu Collaborative Innovation Center on Atmospheric Environment and \\ Equipment Technology (CICAEET), Nanjing University of Information Science \& \\ Technology, Nanjing 210044, China \\ *Corresponding author: jsnanophotonics@yahoo.com
}

\begin{abstract}
:
We demonstrate the excitation of surface phonon polaritons ( $\mathrm{SPhPs}$ ) in the mid-infrared (mid-IR) Reststrahlen band $(10.288 \mu \mathrm{m}-12.563 \mu \mathrm{m})$ on patterned surfaces with silicon carbide $(\mathrm{SiC})$ substrate and gold $(\mathrm{Au})$ gratings. The very large negative permittivity of $\mathrm{Au}$ limits its applications in the mid-IR range, to couple incident light to SPhPs modes, their momentum mismatch can be compensated by patterning Au grating onto the surface of SiC substrate. Samples were fabricated and characterized experimentally by the Fourier transform infrared reflection (FTIR). The optical properties were also simulated by the rigorous coupled wave analysis (RCWA) method. Reflection dips are observed for light polarized vertical to the grating lines (TM-polarized), which are attributed to the coupling of electromagnetic (EM) waves into the SPhP modes. In addition, we present small-volume index sensing with analyte specificity based on mid-IR SPhPs in the fabricated configuration.
\end{abstract}

Keywords: Surface phonon polaritons (SPhPs); Gold gratings; Mid-infrared (Mid-IR); Rigorous coupled wave analysis (RCWA)

PACS: 42.70.Gi; 78.20.Bh; 42.79.Dj; 79.60.Jv 


\section{Introduction}

Strong enhancement of the electromagnetic (EM) field, subwavelength scale confinement, sensitive to changes in the environment and optical non-linearity are among the remarkable effects related to surface plasmons (SPs) [1, 2].These remarkable effects bring many advancements, such as subwavelength waveguiding and modulating, light trapping in solar cells, superlensing, near-field optical microscopy, tip and surface-enhanced Raman scattering, analysis for the label-free detection, as well as enhanced quantum efficiency for detectors in the ultraviolet (UV), visible (VIS) and near-infrared (NIR) spectral ranges [3-15]. Surface plasmon polaritons (SPPs), evanescently confined in the perpendicular direction, are EM excitations propagating at the interface between a dielectric and a conductor. These EM surface waves arise via the coupling of the EM fields to oscillations of the conductor's electron plasma. SPPs can be excited by illuminating the surface under the conditions that provide momentum-matching, called surface plasmon resonance (SPR) conditions, which is shown as a dip in the reflectance spectrum [3]. When the surface mode is excited, the EM energy comes to be strongly confined in the proximity of the surface, with the fields evanescently probing to the surrounding environment. Any change in the refractive index (RI) of outer medium has an effect on varying the propagation constant of SPPs, making it naturally to be exploited as probes for surface analysis and sensing devices. The metal-based plasmonics has been successfully demonstrated in the sensing application with high performance from UV 
to NIR, however, their very large negative permittivities at longer wavelengths limit their usefulness beyond the NIR [16-18].

Fortunately, polar dielectrics offer an opportunity to simultaneously achieve sub-diffraction confinement, low optical losses and operation in the mid-IR to $\mathrm{THz}$ spectral ranges through the stimulation of $\mathrm{SPhP}$ mode, which is the coupling between the EM fields and optical phonons (lattice vibrations) of the polar crystal [19-25]. A high reflectivity and negative real part of permittivity are also observed for polar dielectric crystals within a spectral range referred to as the Reststrahlen band which gives rise to the SPhP phenomenon [26-30]. Within this spectral band, the reflection of incident light approaches to $100 \%$, but unlike that in metals, this is realized without free carriers. SPhPs are studied less extensively than metal-based SPPs due to the fact that few materials can support $\mathrm{SPhPs}[31]$.

Due to the low intrinsic loss, $\mathrm{SPhPs}$, especially on silicon carbide (SiC) based structures, examples of various applications including enhanced near-field light-matter interaction [32], high-sensitivity mid-infrared index sensing [23, 33, 34], mid-IR perfect absorption [35], extraordinary optical transmission and absorption in perforated membranes [36] have been reported. Similar to the case of SPPs, there exists a momentum mismatch between the incident photons and the $\mathrm{SPhP}$ modes, which can be overcame by using the coupling of the incident field to surface modes through the diffraction gratings [20, 24] and high-index prism [21, 37]. The existence of magnetic polariton within the phonon absorption band in SiC-based slit array and deep gratings has been revealed by Z. M. Zhang group [38], and the corresponding 
applications as an IR filter and a coherent thermal emission source were considered.

In this paper, we theoretically describe and experimentally demonstrate the coupling of free-space radiation to $\mathrm{SPhPs}$ on $\mathrm{Au}$ gratings with $\mathrm{SiC}$ substrate. The TM-polarized infrared attenuated total reflection measurements are performed by using transform infrared (FTIR) spectroscopy. Based on the RCWA calculations, the mechanisms of the observed modes are revealed, which offers insights into the anomalous response of the variation of the geometrical parameters. Furthermore, a reflection-type sensor based on an angle scanning method was achieved. The proposed concept can be extended to different wavelength region by using other polar

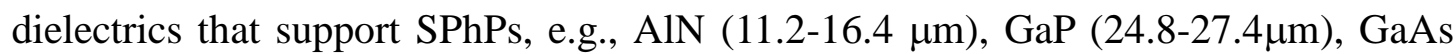
(34.2-37.2 $\mu \mathrm{m})$, CdTe (59.1-70.9 $\mu \mathrm{m})$, and PbSe (49.2-256.5 $\mu \mathrm{m})$ [24].

\section{Structure and theoretical calculation}

The majority of SPhP-based phenomena have been investigated on SiC surfaces because the high-energy phonons of $\mathrm{SiC}$ lie in the mid-IR and are thus accessible using standard IR sources and detectors [19, 21, 22]. In the absence of free carriers, the frequency-dependent dielectric permittivity of $\mathrm{SiC}$ is given by: [39, 40]

$$
\varepsilon_{\mathrm{SiC}}(\omega)=\varepsilon_{\mathrm{SiC}}^{\prime}+i \varepsilon_{\mathrm{SiC}}^{\prime \prime}=\varepsilon_{\infty} \frac{\omega^{2}-\omega_{\mathrm{LO}}^{2}+i \gamma \omega}{\omega^{2}-\omega_{\mathrm{TO}}^{2}+i \gamma \omega}
$$

where the longitudinal optical phonon frequency $\omega_{\mathrm{LO}}=972 \mathrm{~cm}^{-1}$ at $\lambda_{\mathrm{LO}}=10.288 \mu \mathrm{m}$, the transverse optical phonon frequency $\omega_{\mathrm{TO}}=796 \mathrm{~cm}^{-1}$ at $\lambda_{\mathrm{TO}}=12.563 \mu \mathrm{m}$, the damping rate due to vibrational anharmonicity $\gamma=3.75 \mathrm{~cm}^{-1}$, and $\varepsilon_{\infty}=6.5$. The Reststrahlen band defined by $\varepsilon_{\mathrm{sic}}^{\prime}<0$ corresponds to $\omega_{\mathrm{TO}}<\omega<\omega_{\mathrm{LO}}$, within which $\mathrm{SPhPs}$ can be excited. In order to couple the incident light to $\mathrm{SPhP}$ modes, their 
momentum mismatch can be bridged by patterning a grating onto the materials' interface $[20,24,38]$.
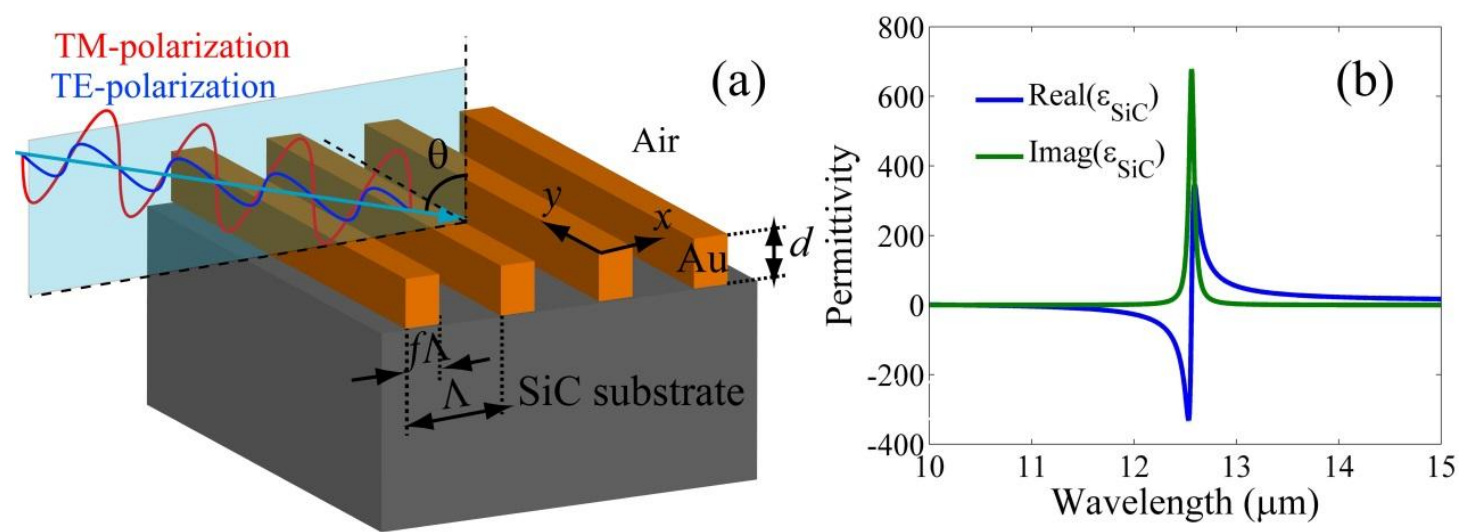

Fig.1. (a) The schematic image of the Au grating patterned on the SiC substrate. The angle $\theta$ denotes the incident angle to the sample surface. (b) Real (blue) and imaginary (green) parts of the permittivity of SiC around its Reststrahlen band.

The structure used and coordinates system in the calculations are shown in Fig. 1 (a), where the $x$-direction is parallel to the surface and perpendicular to the ridges of the grating. The $y$-direction is assumed to be infinite along the grating grooves in this study, the $z$-direction is set along the surface normal, and $\theta$ represents the angle of incidence (from normal). The permittivity of $\mathrm{SiC}$ was modeled using the equation developed in Ref. 40 and is shown in Fig. 1(b). The simulation of optical phonons within the Reststrahlen band of SiC induces a negative permittivity which is similar to the plasmonic materials below their plasma frequency. Outside of this band, SiC behaves as a typical and transparent dielectric material mainly defined by its index of refraction.

Two-dimensional rigorous coupled-wave analysis (RCWA) algorithm [41-43], a frequency domain technique which utilizes the Floquet theorem to calculate the 
amplitudes of a large number of spectral orders of diffraction is employed to study the spectral property of the proposed structure with a plane wave incident on the grating. Although no approximations to Maxwell's equations are made in the RCWA method, the number of spatial harmonics retained in calculations must be truncated in order to obtain a solution. For 2D grating problems, the number of equations to be solved increases with the square of the number of orders retained, and the tradeoff between speed and accuracy becomes much severer. To ensure the numerical accuracy of the calculation with reasonable computational time, all the calculations have been tested for convergence and calculated with the number of diffracted orders equal to 203.

The frequency-dependent dielectric constant of Au is expressed by Lorentz-Drude model [44]:

$$
\varepsilon_{\mathrm{Au}}(\omega)=\varepsilon_{\infty}-\frac{\omega_{p}^{2}}{\omega^{2}+i \Gamma_{p} \omega}-\frac{f_{1} \omega_{1}^{2}}{\omega^{2}-\omega_{1}^{2}+i \Gamma_{1} \omega}
$$

where $\omega_{\mathrm{p}}$ and $\omega$ are the frequencies of the plasma and interest respectively, $\Gamma_{\mathrm{p}}$ is the damping (or relaxation) rate, $i$ denotes the complex number $(i=\sqrt{-1}$ ), and the contribution from interband transitions at infinite frequency is $\varepsilon_{\infty}$. The Lorentz term includes the Lorentz oscillator damping rate $\Gamma_{1}$, the Lorentz resonance width $\omega_{1}$ and the weighting factor $f_{1}$. The values of different parameters for Au are given in Table 1.

Table 1. Values of Lorentz-Drude parameters for $\mathrm{Au}$

\begin{tabular}{ll}
\hline Parameters & Values \\
\hline
\end{tabular}




\begin{tabular}{cc}
\hline$\varepsilon_{\infty}$ & 6.8890 \\
$\omega_{\mathrm{p}}(\mathrm{ev})$ & 8.9601 \\
$\omega_{1}(\mathrm{ev})$ & 2.9715 \\
$\Gamma_{\mathrm{p}}(\mathrm{ev})$ & 0.0723 \\
$\Gamma_{1}(\mathrm{ev})$ & 0.9503 \\
$f_{1}$ & 1.7857 \\
\hline
\end{tabular}

Reflectance of the designed sample as a function of grating period and incident wavelength is shown in Fig. 2(a), where only the zero order mode is propagating and the other modes are evanescent. The parameters are chosen as $f=0.22, d=0.28 \mu \mathrm{m}$, and $\theta=45^{\circ}$. It is understood that the dark area is caused by propagating $\mathrm{SPhP}$ which is generated by different diffracted orders from the grating. Or in other words, the $k$-vector of a diffracted order for normal incidence equals to an integer multiple of the grating vector. As a $\mathrm{SPhP}$ wave propagates at the interface, the conservation of wave vector is provided by diffraction on a periodic structure [3]: 

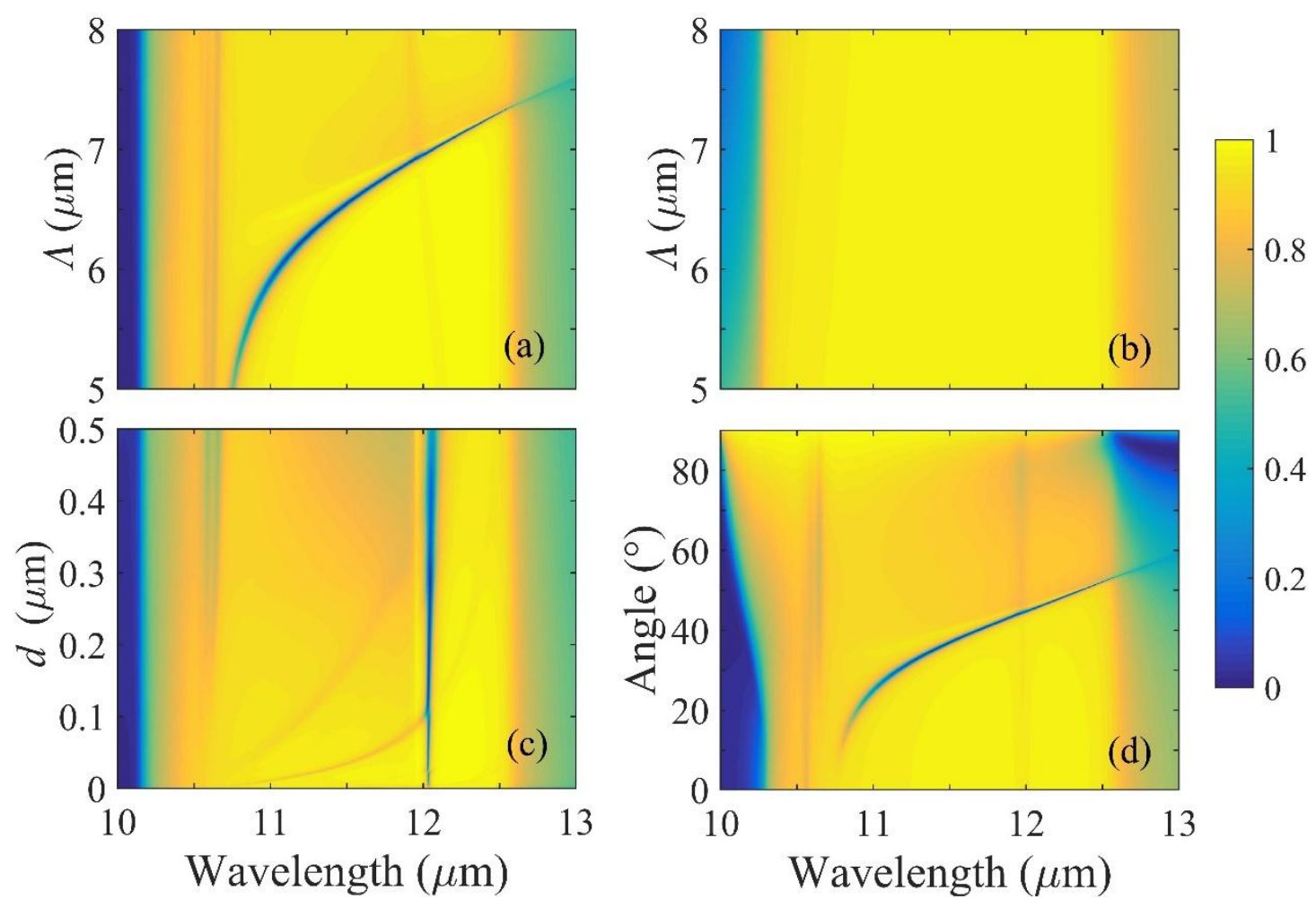

Fig. 2. Reflectance as functions of wavelength and grating period $\Lambda$ (a), grating thickness $d$ (c) and incident angle $\theta(\mathrm{d})$. (b) Zero order reflectance versus $\Lambda$ and wavelength with normal incidence, the other parameters are the same as used in Fig. 2 (a). The color scale shows reflectivity from 0 to 1 .

$$
\mathbf{k}_{\mathrm{SPhP}}=\mathbf{k}_{0} \sin \theta \pm i\left(\frac{2 \pi}{\Lambda_{x}}\right) \mathbf{u}_{x} \pm i\left(\frac{2 \pi}{\Lambda_{y}}\right) \mathbf{u}_{y}
$$

where $\mathbf{k}_{\mathrm{SPhP}}$ indicates the $\mathrm{SPhP}$ wavevector, $\mathbf{k}_{0}$ denotes the wavevector of light, $\theta$ is the angle of incidence, $\Lambda_{\mathrm{x}}, \Lambda_{\mathrm{y}}$ are the periodicity of the structured film in the $x$-and $y$-directions respectively, $\mathbf{u}_{x}$ and $\mathbf{u}_{y}$ are the reciprocal lattice vectors, while $i$ and $j$ are integer numbers corresponding to different directions of SPhP. In addition, the magnitude of SPhP wave vector is given by

$$
\left|\mathbf{k}_{\mathrm{SPhP}}\right|=\frac{2 \pi}{\lambda_{0}} \sqrt{\frac{\varepsilon_{\mathrm{SiC}\left(\lambda_{0}\right)} \varepsilon_{\mathrm{d}}}{\varepsilon_{\mathrm{SiC}\left(\lambda_{0}\right)}+\varepsilon_{\mathrm{d}}}}
$$

where $\lambda_{0}$ is the wavelength of incident light, $\varepsilon_{\mathrm{SiC}(\lambda 0)}$ is described as the complex permittivity of $\mathrm{SiC}$, and $\varepsilon_{\mathrm{d}}$ means the permittivity of the adjacent medium. For 
comparison, the reflectivity of the grating structure with the same parameters used in Fig. 2(a) is calculated and shown in Fig. 2(b). Obviously, within the Reststrahlen band, the real part of $\varepsilon_{\mathrm{SiC}}$ is negative, leading to a dramatic increase in reflectivity for TE-polarization.

SPhPs can be generated by the diffracted orders of light scattered from a metallic grating [45]. This scattered radiation has a larger momentum than that of the incident radiation by an integer number of reciprocal lattice vectors. To better understand the SPhP phenomenon, we plot the reflectivity contour map versus wavelength and grating thickness with the electric field parallel to the grating vector, as depicted in Fig. 2(c). In practical calculation, the adopted parameters are same as those used in Fig. 2(a) except for the thickness of grating (d). A fascinatingly well-behaved independence on grating thickness has been revealed. Usually, the spectral response of grating-based devices is highly dependent on the structural parameters [46]. It is difficult to fabricate an ideal structure with the same parameters as the designed one. During the process of grating fabrication, the most difficult thing is to control the thickness. Thus, we evaluate the response of the structure under variation in the grating thickness. Keeping other parameters as constants, it is found that the resonant wavelength almost does not change for $d<0.5 \mu \mathrm{m}$. Therefore, this type of grating is much easier to achieve compared with previous designs. Figure 4(d) shows that the SPhP only exists for large incident angles. The grating structure was assigned the following parameters: $f=0.22, d=0.28 \mu \mathrm{m}$, and $\Lambda=7 \mu \mathrm{m}$. Under the situation with small $\theta$, the evanescent wave emerging from the grating is very weak 
when it arrives at the $\mathrm{SiC}$, so there is little observable $\mathrm{SPhP}$ excitation.

\section{Experiments}

The preparation of substrates must be extremely careful. After cleaning in a mixed solution containing hydrochloric acid, hydrofluoric acid, and deionized water with the a mixing ration (volume ratio) of 1:1:4, the substrate was dried by $\mathrm{N}_{2}$ and baked in quartz tube at $150^{\circ} \mathrm{C}$ for $1 \mathrm{~h}$. Chrome $(\mathrm{Cr})$ thin films with a thickness of $10 \mathrm{~nm}$ was deposited and used as interlayer between $\mathrm{SiC}$ wafer and gold film to promote adhesion and decrease the internal stresses. Gold film with a thickness of $280 \mathrm{~nm}$ was also deposited using electron beam evaporation. The system was pumped to a base pressure of $4 \times 10^{-6} \mathrm{mbar}$ at $20^{\circ} \mathrm{C}$. The films were deposited by using electron beam evaporation under the oxygen partial pressure of $5 \times 10^{-4} \mathrm{mbar}$ at $0.1 \mathrm{~nm} / \mathrm{s}$. Then the silicon wafers were patterned by electron beam lithography on AZ5214 photoresist (with a thickness of about $1.5 \mu \mathrm{m}$ ) with the inverse pattern of the desired grating. The sample was baked at $85^{\circ} \mathrm{C}$ for 30 mins. The plasma of a $\mathrm{CF}_{4} / \mathrm{Ar}$ gas mixture was applied to the grating-patterned surface for $12-18 \mathrm{~min}$ at room temperature to leave the grating nanolines through removal of the metallic coating. The plasma power, chamber pressure, and the gas flow rates were adjusted to $100 \mathrm{~W}, 10 \mathrm{~Pa}$, and $30 \mathrm{sccm}$ and $5 \mathrm{sccm}$, respectively. $\mathrm{O}_{2}$ plasma was then applied to the top surface to expose the head portion of the Au grating. The plasma power, the gas flow rate and etching time

were tuned to $150 \mathrm{~W}, 30 \mathrm{sccm}$ and $5.5 \mathrm{mins}$, respectively. Figure 3 presents a micrograph by scanning electron microscopy (SEM) for the fabricated gold grating. 


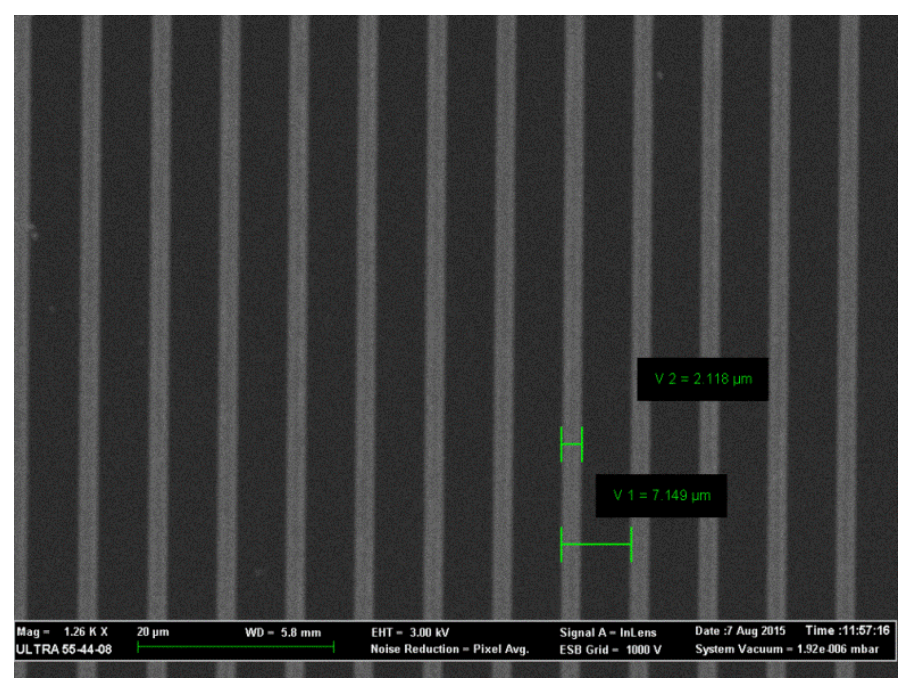

Fig. 3. A SEM micrograph for the fabricated gold grating on a SiC substrate.

Reflection spectra were measured with a Bruker V80v Fourier transform infrared (FTIR) vacuum spectrometer by using an internal room-temperature pyroelectric deuterated triglycine sulfate far-infrared detector [24]. The experiment uses a PIKE Technologies 10Spec specular reflection accessory, giving reflection spectra at an angle of incidence of $10^{\circ}$ within the vacuum sample compartment, thus minimizing effects of atmospheric absorption. The KRS-5 far-IR beam-splitter was used to select the polarization of the incident light. SPhPs can only be excited by incident light with a component of the electric field perpendicular to the grating ridge. All reflection data were normalized to the reflection through a gold mirror. 


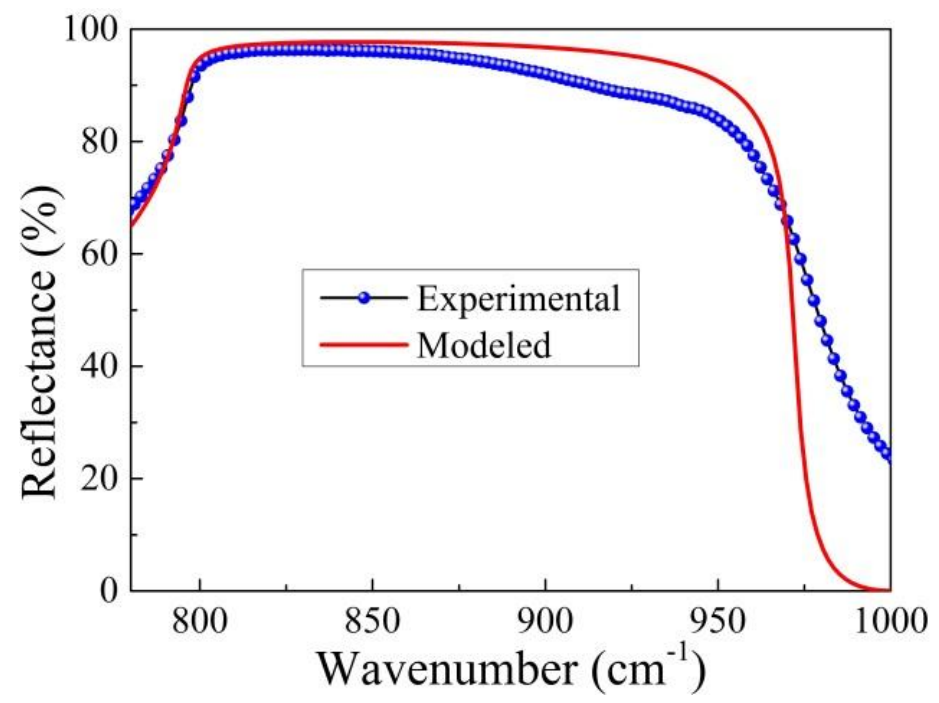

Fig.4. Modeled (red, calculated from the permittivity in Eq.1) and experimental (blue diamond) reflection from a flat $\mathrm{SiC}$ surface.

Using the permittivity described in Eq.1, the reflectivity from a flat SiC surface is calculated for TE and TM- polarized light at a zero angle of incidence. The calculated and experimental reflection spectra (TM-polarized) are shown in Fig. 4 and they agree well with each other. It is noted that the experimental reflection somewhat smaller than the predicted reflection in short wavelength region of the "Reststrahlen" band, perhaps due to either (i) imperfect reflection from the $\mathrm{Au}$ mirror used for normalization or alternatively (ii) the difficulty in fitting the highly dispersive portion of the SiC permittivity using Eq.1.

A TM-polarized plane wave was launched within the $x z$-plane at an incident angle of $45^{\circ}$ in the simulation to approximate the experimental conditions. A typical pair of reflection spectra from the experiment and RCWA calculation are shown in Fig.5 with diameters of $\Lambda=7.149 \mu \mathrm{m}, h=0.28 \mu \mathrm{m}$ and $f=0.296$. The measured reflection spectrum displays double pronounced minima, which do not appear in the transmission spectrum for the case of the plane structures. These dips in the reflection are due to 
the excited phonon resonances. The differences between the experimental and simulated spectra can be ascribed to the additional induced surface damage caused by the reactive-ion etching process during the experiment. Such a discrepancy could be an indication of the difficulty in accurately modeling the $\mathrm{SiC}$ permittivity closer to the phonon resonance [19]. In addition, this discrepancy could also result in a greater sensitivity of our response, in this frequency region, even to slight changes in beam collimation, incidence angle, or sample position $[19,24]$. Although the intrinsic optical losses associated with the fast scattering lifetimes pose an inevitable limitation on the performance of metals in the mid-IR wavelength range, the occurrence of the negative permittivity within the underlying $\mathrm{SiC}$ substrate results in the observation of dips.

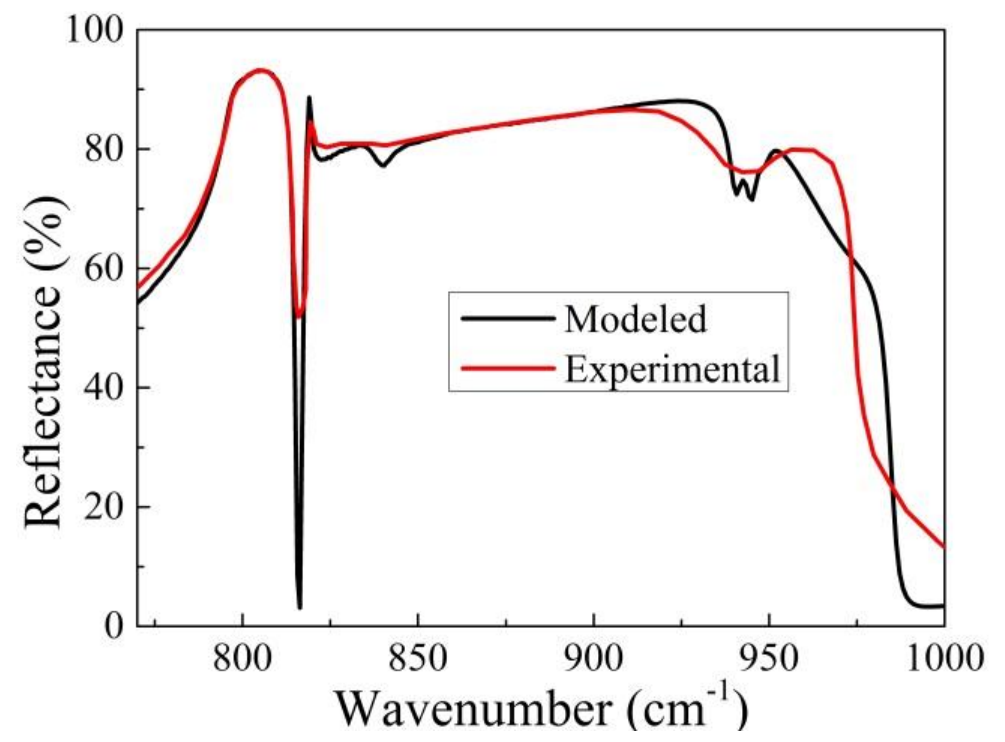

Fig. 5. Experimental and modeled reflection spectra from patterned Au grating on $\mathrm{SiC}$ substrate.

In addition, to experimentally demonstrate SPhPs, the FTIR measurements were used to detect the resonant angles $\left(\theta_{\text {res }}\right)$. All scans of wavenumber display minimal $R$ and maximal SPhP excitation, which are shown in Fig. 6. As can be seen, $\theta_{\text {res }}$ shifts 
toward a smaller angle as the wavenumber increases. Another important quantity to describe the quality of the $\mathrm{SPhP}$ is the $\mathrm{Q}$ factor $\left(\omega_{0} / \mathrm{FWHM}\right.$, full-width at half maximum) of its resonances [47]. The $\mathrm{Q}$ factor of $\mathrm{SPhP}$ resonance is larger than that of the dipole-like slot antenna resonance $(\mathrm{Q} \sim 10-20)$, Mie scattering $(\mathrm{Q} \sim 10-30)$ of the $\mathrm{SiC}$ whiskers [28]. We also notice that the $\mathrm{Q}$ factor of our $\mathrm{SPhP}$ resonance is higher compared to other theoretically proposed localized SPhP resonances $(\mathrm{Q} \sim 50-60)$ of the SiC nanodisks [48] and in the same order as the localized $\mathrm{SPhP}$ resonance (Q 40-135) of the $\mathrm{SiC}$ pillar arrays [49].

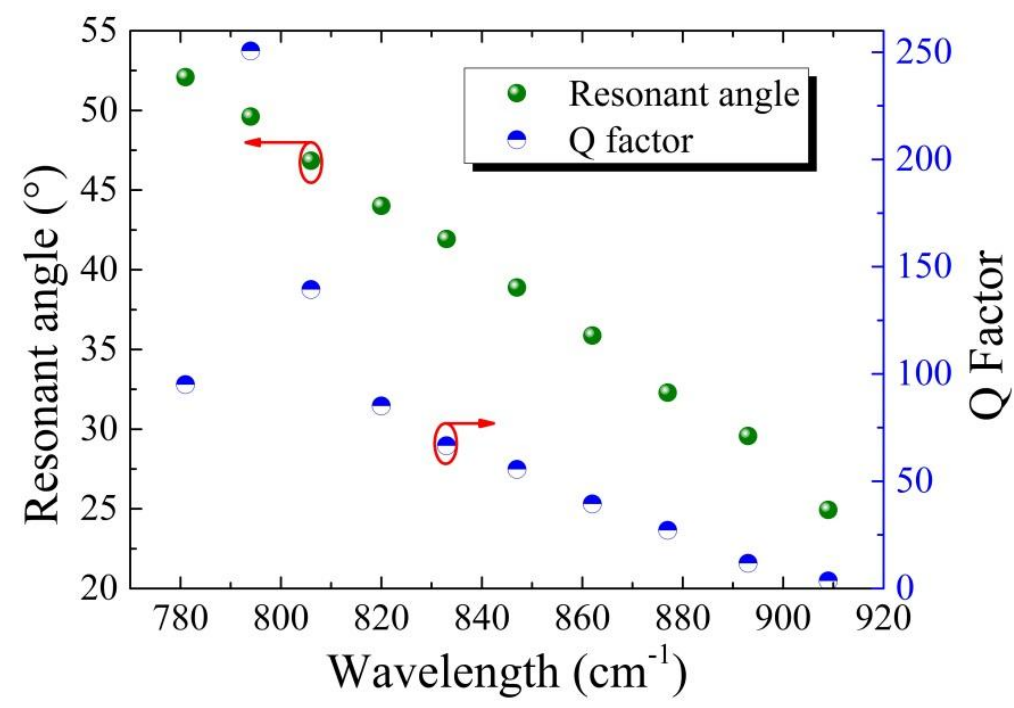

Fig. 6. Experimental plots of resonant angle and Q factor versus wavenumber.

\section{Potential sensing applications}

Molecules exhibit unique spectroscopic signatures in the mid-IR fingerprint region that are manifested with larger index $(n)$ differences compared to materials operating in the visible range [23]. The requirement of small-volume sensitivity explains the widespread use of SPPs. SPhPs hold great potential for small-volume mid-IR sensing because of the high sensitivity to analyte permittivity $\left(\varepsilon_{\mathrm{a}}=n_{\mathrm{a}}{ }^{2}\right)$. 
The change in the resonance curve caused by a change in the RI $\Delta n$ can be characterized either by an angular shift of the curve $\Delta \theta_{\text {res }}$ (sensing by angular modulation) or a change in the reflectance $\Delta \mathrm{R}$ at a fixed angle (sensing by intensity modulation). According to previous papers on the performance of SPR sensors [50, 51], sensitivity by intensity is expressed by:

$$
S_{I}(\theta)=\lim _{\Delta n \rightarrow 0} \frac{\Delta R(\theta)}{\Delta n}=\frac{\partial R(\theta)}{\partial n}
$$

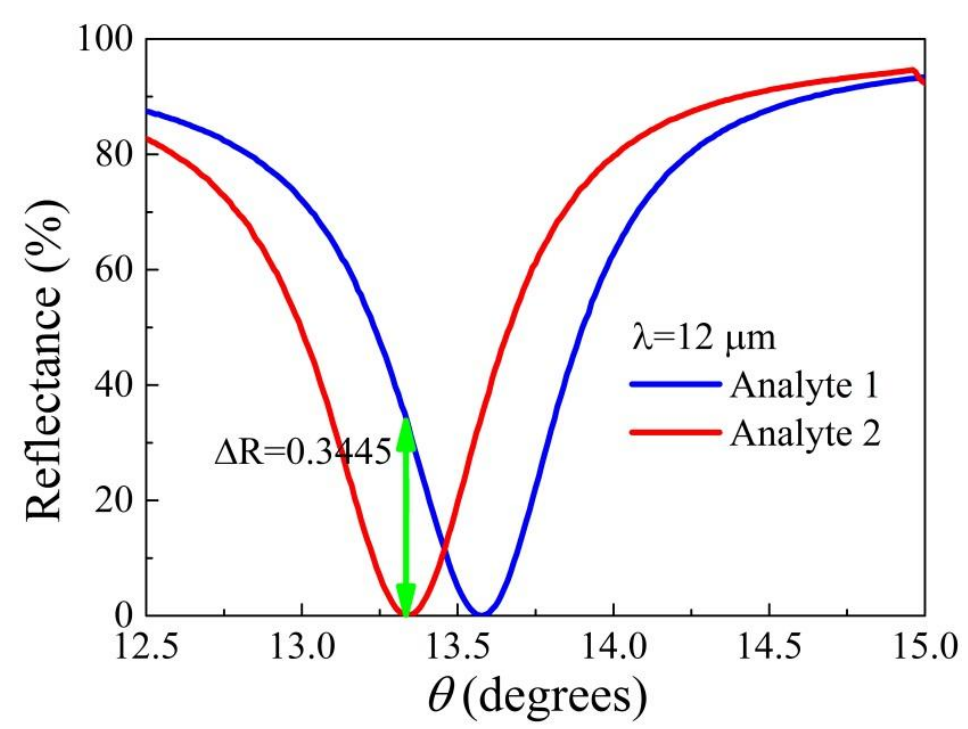

Fig. 7. Theoretical reflectivity with $2 \mu \mathrm{m}$ of analytes 1,2 contained on the top of the structure.

We theoretically investigate a reflection-type sensor based on an angle scanning method. The sensing element consists of Au grating embedded in a SiC substrate in which excitation of SPhPs was achieved by illumination with TM-polarized light. We characterized the sensor response by the RI changes in the environment. Using a wavelength of $\lambda=12 \mu \mathrm{m}$, we calculated the reflectivities for analytes $1(n=1.33)$ and 2 $(n=1.334)$, which are shown in Fig. 7. Here, $\Delta n=0.004$ RIU, but $R$ increases by $34.45 \%$. The ratio of $\Delta R / \Delta n$ is $86.125 \mathrm{RIU}^{-1}$ for the $\mathrm{SPhR}$ sensor. The value suggests at least qualitatively that the present sensor offers a high sensitivity by intensity. 
Similar to SPPs, SPhPs can only be excited through evanescent field coupling under TM-polarization. In order to ensure evanescent field coupling, the obvious choices can be employed either Kretschmann configuration or Otto configuration or a grating $[20,21,24,35,40]$. The Kretschmann configuration requires thin film deposition on a high index prism. The quality of resonance response is quite poor and the property of the phononic material is dependent on the deposition conditions, such as temperature and pressure. For the Otto configuration, the method does not require thin film deposition, however, the results published so far do not show as good a phononic response as expected. Another method is to employ a grating to increase the $k$-vector of the incident photons, which can be achieved by electron beam lithography patterning directly onto the phononic material surface as the authors did in this work.

\section{Conclusion}

We have experimentally demonstrated excitation of SPhPs in the mid-IR range on patterned structures with $\mathrm{SiC}$ substrate and Au gratings. The presence of the negative permittivity substrate $(\mathrm{SiC})$ enables the stimulation of $\mathrm{SPhPs}$. The $\mathrm{SPhP}$ mode supported in the fabricated structures was studied through both the FTIR spectroscopy measurements and numerical simulations. The results show the spectral dependence of the SPhP mode on the geometry. The TM-polarized spectrum shows two prominent reflection dips at 820 and $948 \mathrm{~cm}^{-1}$, the experimental observations are in good agreement with the RCWA predicted results. Finally, small-volume index sensing with analyte specificity based on mid-IR SPhPs in the fabricated configuration is also presented. It is important to note that this method is not limited to SiC-based SPhPs, 
but also applicable to other types of polar dielectrics.

\section{Acknowledgements}

This work is supported by National Science Foundation of China (NSFC) (61203211, 41675154); the Natural Science Foundation of the Jiangsu Province (BK20141483) and the Six Major Talent Peak expert of Jiangsu Province (2015-XXRJ-014).

\section{References}

[1] H. Rather, Surface plasmons, Berlin, Springer-Verlag Press, 1988.

[2] S. A. Maier, Plasmonics: fundamentals and applications, Bath, Springer Press, 2007.

[3] L. Novotny B. Hecht, Principles of nano-optics, New York, Cambridge University Press, 2006.

[4] H. A. Atwater, A. Polman, Plasmonics for improved photovoltaic devices, Nature Mater. 9 (2010) 205-213.

[5] S. Lal, S. Link, N. J. Halas, Nano-optics from sensing to waveguiding, Nature Photon. 1 (2007) 641-648.

[6] J. D. Caldwell, O. J. Glembocki, F. J. Bezares, N. D. Bassim, R. W. Rendell, M. Feygelson, M. Ukaegbu, R. Kasica, L. Shirey, C. Hosten, Plasmonic nanopillar arrays for large-area, high-enhancement surface-enhanced raman scattering sensors, ACS Nano 5 (2011) 4046-4055.

[7] L. L. Jin, G. W. She, J. Li, J. Xia, X. T. Wang, L. X. Mu, W. S. Shi, A facile 
fabrication of $\mathrm{Ag}-\mathrm{Au}-\mathrm{Ag}$ nanostructures with nanogaps for intensified surface-enhanced Raman scattering, Appl. Surf. Sci. 389 (2016) 67-72

[8] A. Aubry, D. Y. Lei, A. I. Fernandez-Dominguez, Y. Sonnefraud, S. A. Maier, J. B. Pendry, Plasmonic light-harvesting devices over the whole visible spectrum, Nano Lett. 10 (2010) 2574-2579.

[9] W. L. Barnes, A. Dereux, T. W. Ebbesen, Surface plasmon subwavelength optics, Nature 424 (2003) 824-830.

[10] C. Clavero, Plasmon-induced hot-electron generation at nanoparticle/metal-oxide interfaces for photovoltaic and photocatalytic devices, Nature Photon. 8 (2014) 95-103.

[11] A. Sobhani, M. W. Knight, Y. Wang, L. V. Brown, Z. Fang, P. Nordlander, N. J. Halas, Narrowband photodetection in the near-infrared with a plasmon-induced hot electron device, Nature Commun. 4 (2013) 1643.

[12] I. Goykhman, B. Desiatov, J. B. Khurgin, J. Shappir, U. Levy, Locally oxidized silicon surface-plasmon schottky detector for telecom regime, Nano Lett. 11 (2011) 2219-2224.

[13] R. Jolly Bosea, V.S. Kavithaa, C. Sudarsanakumarb, V. P. Mahadevan Pillai, Phase modification and surface plasmon resonance of Au/WO3 system, Appl. Surf. Sci. 379 (2016) 505-515.

[14] L. J. Yan, Y. N. Yan, L. L. Xu, R. R. Ma, F. X. Jiang, X. H. Xu, Large range localized surface plasmon resonance of Ag nanoparticles films dependent of surface morphology, Appl. Surf. Sci. 367 (2016) 563-568. 
[15] Shweta Verma, B. Tirumala Rao, S. Bhartiya, V. Sathe, L. M. Kukrej, Growth temperature dependent surface plasmon resonances of densely packed gold nanoparticles' films and their role in surface enhanced Raman scattering of Rhodamine6G, Appl. Surf. Sci. 346 (2015) 379-387.

[16] P. R.West, S. Ishii, G. V. Naik, N. K. Emani, V. M. Shalaev, A. Boltasseva, Searching for better plasmonic materials, Laser Photon. Rev. 4 (2010) 795-808.

[17] A. Boltasseva, H. A. Atwater, Low-loss plasmonic metamaterials, Science 331 (2011) 290-291.

[18] J. B. Khurgin, G. Sun, Scaling of losses with size and wavelength in nanoplasmonics and metamaterials, Appl. Phys. Lett. 99 (2011) 211106.

[19] Y. Chen, Y. Francescato, J. D. Caldwell, V. Giannini, T. W. W. Maß, O. J. Glembocki, F. J. Bezares, T. Taubner, R. Kasica, M. Hong, S. A. Maier, Spectral tuning of localized surface phonon polariton resonators for low-loss mid-IR applications, ACS Photonics 1 (2014) 718-724.

[20] A. J. Huber, B. Deutsch, L. Novotny, R. Hillenbrand, Focusing of surface phonon polaritons, Appl. Phys. Lett. 92 (2008) 203104.

[21] S. S. Ng, Z. Hassan, H. Abu Hassan, Surface phonon polariton of wurtzite GaN thin film grown on c-plane sapphire substrate, Solid State Commun. 145 (2008) $535-538$.

[22] N. Ocelic, R. Hillenbrand, Subwavelength-scale tailoring of surface phonon polaritons by focused ion-beam implantation, Nature Mater. 3 (2004) 606-609.

[23] B. Neuner, D. Korobkin, C. Fietz, D. Carole, G. Ferro, G. Shvets, Midinfrared 
index sensing of pL-scale analytes based on surface phonon polaritons in silicon carbide, J. Phys. Chem. C 114 (2010) 7489-7491.

[24] W. Streyer, S. Law, A. Rosenberg, C. Roberts, V. A. Podolskiy, A. J. Hoffman, D. Wasserman, Engineering absorption and blackbody radiation in the far-infrared with surface phonon polaritons on gallium phosphide, Appl. Phys. Lett.104 (2014) 131105.

[25] Giuseppe D'Aguanno, Coherent thermal emission near $10.6 \mu \mathrm{m}$ mediated by localized phonon-polariton modes in microparticle arrays, Appl. Phys. Lett. 106 (2015) 031110.

[26] J. Le Gall, M. Olivier, J. J. Greffet, Experimental and theoretical study of reflection and coherent thermal emissionby a SiC grating supporting a surface-phonon polariton, Phys. Rev. B 55 (1997) 10105.

[27] J. Greffet, R. Carminati, K. Joulain, J. Mulet, S. Mainguy, Y. Chen, Coherent emission of light by thermal sources, Nature 416 (2002) 61-64.

[28] J. A. Schuller, T. Taubner, M. L. Brongersma, Optical antenna thermal emitters, Nature Photon. 3 (2009) 658-661.

[29] B. Neuner III, C. Wu, G. T. Eyck, M. Sinclair, I. Brener, G. Shvets, Efficient infrared thermal emitters based on low-albedo polaritonic meta-surfaces, Appl. Phys. Lett. 102 (2013) 211111.

[30] J. A. Schuller, R. Zia, T. Taubner, M. L. Brongersma, Dielectric metamaterials based on electric and magnetic resonances of silicon carbide particles, Phys. Rev. Lett. 99 (2007) 107401. 
[31] J. D. Caldwell, L. Lindsay, V. Giannini, I. Vurgaftman, T. L. Reinecke, S. A. Maier, O. J. Glembocki, Low-loss, infrared and terahertz nanophotonics using surface phonon polaritons, Nanophotonics 4 (2015) 44-68.

[32] R. Hillenbrand, T. Taubner, F. Keilmann, Phonon-enhanced light-matter interaction at the nanometre scale, Nature 418 (2002) 159-162.

[33] I. Balin, N. Dahan, V. Kleiner, E. Hasman, Slow surface phonon polaritons for sensing in the midinfrared spectrum, Appl. Phys. Lett. 94 (2009) 111112.

[34] G. G. Zheng, Y. Y. Chen, L. B. Bu, L. H. Xu, W. Su, Waveguide-coupled surface phonon resonance sensors with super-resolution in the mid-infrared region, Opt. Lett. 41 (2016) 1582-1585.

[35] G. C. R. Devarapu, S. Foteinopoulou, Mid-IR near-perfect absorption with a SiC photonic crystal with angle-controlled polarization selectivity, Opt. Express 20 (2012) 13040-13054.

[36] D. Korobkin, Y. A. Urzhumov, B. Neuner III, C. Zorman, Z. Zhang, I. D. Mayergoyz, G. Shvets, Mid-infrared metamaterial based on perforated SiC membrane: engineering optical response using surface phonon polaritons, Appl. Phys. A 88 (2007) 605-609.

[37] B. Lahiri, G. Holland, V. Aksyuk, A. Centrone, Nanoscale imaging of plasmonic hot spots and dark modes with the photothermal induced resonance technique, Nano Lett. 13 (2013) 3218-3224.

[38] L. P. Wang, Z. M. Zhang, Phonon-mediated magnetic polaritons in the infrared region, Opt. Express 19 (2011) A126-A135. 
[39] E. D. Palik, Handbook of optical constants of solids, San Diego, Academic Press, 1998.

[40] B. Neuner III, D. Korobkin, C. Fietz, D. Carole, G. Ferro, G. Shvets, Critically coupled surface phonon-polariton excitation in silicon carbide, Opt. Lett. 34 (2009) 2667-2669.

[41] S. S. Wang, R. Magnusson, Theory and applications of guided-mode resonance filters, Appl. Opt. 32 (1993) 2606-2613.

[42] X. Y. Fu, K. Yi, J. D. Shao, Z. X. Fan, Nonpolarizing guided-mode resonance filter, Opt. Lett. 34 (2009) 124-126.

[43] D. L. Brundrett, E. N. Glytsis, T. K. Gaylord, Normal-incidence guided-mode resonant grating filters: design and experimental demonstration, Opt. Lett. 23 (1998) 700-702.

[44] P. B. Johnson, R. W. Christy, Optical constants of the noble metals, Phys. Rev. B 6 (1972) 4370-4379.

[45] J. G. Rivas, G. Vecchi, V. Giannini, Surface plasmon polariton-mediated enhancement of the emission of dye molecules on metallic gratings, New J. Phys. 10 (2008) 105007.

[46] C. Song, X. S. Xia, Z. D. Hu, Y. J. Liang, J. C. Wang, Characteristics of plasmonic Bragg reflectors with graphene-based silicon grating, Nanoscale Res. Lett. 11 (2016) 419.

[47] T. Wang, P. N. Li, B. Hauer, D. N. Chigrin, T. Taubner, Optical properties of single infrared resonant circular microcavities for surface phonon polaritons, 
Nano Lett. 13 (2013) 5051-5055.

[48] M. Ameen, A. García-Etxarri, M. Schnell, R. Hillenbrand, J. Aizpurua, Infrared phononic nanoantennas: Localized surface phonon polaritons in $\mathrm{SiC}$ disks, Chinese Sci. Bull. 55 (2010) 2625-2628.

[49] J. D. Caldwell, O. J. Glembocki, Y. Francescato, N. Sharac, V. Giannini, F. J. Bezares, J. P. Long, J. C. Owrutsky, I. Vurgaftman, J. G. Tischler, V. D. Wheeler, N. D. Bassim, L. M. Shirey, R. Kasica, S. A. Maier, Low-loss, extreme subdiffraction photon confinement via silicon carbide localized surface phonon polariton resonators, Nano Lett. 13 (2013) 3690-3697.

[50] D. V. Nesterenko, Z. Sekkat, Resolution estimation of the $\mathrm{Au}, \mathrm{Ag}, \mathrm{Cu}$, and $\mathrm{Al}$ single- and double-layer surface plasmon sensors in the ultraviolet, visible, and infrared regions, Plasmonics 8 (2013) 1585-1595.

[51] J. Homola, I. Koudela, S. S. Yee, Surface plasmon resonance sensors based on diffraction gratings and prism couplers: sensitivity comparison, Sens. Actuators B 54 (1999) 16-24. 